\title{
The pyramids of Gizeh, reductionist research-based progress, unintended consequences and the complexity of medicine
}

\author{
Bing Siang Gan, MD PhD FRCSC FACS \\ Departments of Surgery and Medical Biophysics, Schulich School of Medicine and Dentistry \\ Western University
}

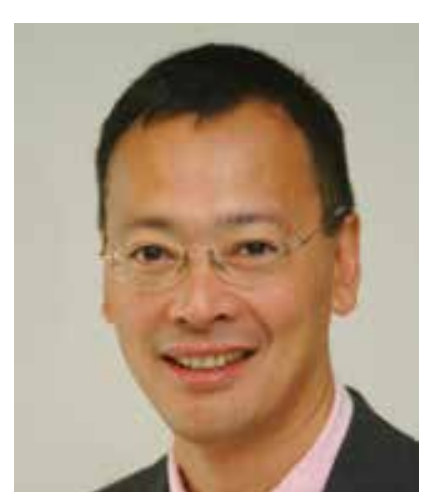

\begin{abstract}
$\mathrm{BIO}$
Bing graduated from the Medical Faculty at Erasmus University in Rotterdam, The Netherlands in 1988. He then completed a PhD in Medical Sciences (University of Calgary), internship (University of Regina) and surgical residency (University of Western Ontario) and post-residency clinical fellowships (University of Toronto and Harvard University) followed by a research post-doctoral fellowship (Department of Cell Biology, University of Toronto).

Bing has been with the Roth | McFarlane Hand and Upper Limb Centre at St. Joseph's Health Centre since 1998. He is a Professor of Surgery and Medical Biophysics at Western University. His clinical practice focuses on hand and wrist surgery, microsurgical reconstruction and complex wound reconstruction, with a particular clinical and research interest in patients with Dupuytren's contracture. He is also interested in other fibrosing conditions, such as hypertrophic scarring. Bing was a Canadian Society for Clinical Investigation (CSCI) Member of Council 2004-2011and CSCI President 2009-2011.
\end{abstract}

\begin{abstract}
$\mathrm{R}$ ecently, I was watching a documentary on the great pyramids of Gizeh. One of the things that stuck in my mind is that it is not known how the ancient Egyptians built these immense structures. It made me think about the paradox that while humanity has achieved immeasurable progress in the more than 4,500 years since the building of these impressive edifices, it has simultaneously lost some remarkable knowledge.
\end{abstract}

\section{Our journey has many turns}

When I started in my career as a surgeon-scientist, now about 25 years ago, I was interested in the field of cell signalling and the molecular control of disease development. As a hand surgeon, I came to focus on a deformity of the hand called Dupuytren's disease, a quirky disorder that leads to finger contractures as a result of the deposition of dense collagenous cords in specific patterns in certain structures of the hand; preventing the fingers from moving. Previously, treatment consisted of tedious surgery to remove these cords, requiring dissec- tion of the fine anatomy of the hand, but this surgery carried a high level of morbidity and required weeks, if not months, of post-operative hand therapy to make the fingers agile again. Despite this intensive post-operative therapy, in a significant percentage of patients, range of motion and hand strength never fully returned.

It is unclear why this collagen cord formation occurs, and over the past two decades our lab has focussed on unravelling the molecular cues underlying this process. In our lab, we examined many different fibrogenic molecular pathways and looked at the response patterns of 
primary Dupuytren's cells to pharmacological manipulation of these pathways, alterations in environment and other stimuli. Our research used well-acceptable scientific principles, where we were able to measure small, but significant molecular changes, using a reductionist approach and keeping as many factors as we could control constant. It allowed us to infer clear cause-effect relationships. Based on our data, and based on the assumption that we could "cure" this disease by reversing some of the observed cause-effect relationships, we inferred tremendous general curative benefits and even implied that our data could potentially be used to alleviate other fibrogenic conditions. The Canadian Institutes for Health Research (CIHR) and other agencies supported these thoughts with many grants and awards.

We presented our work at many scientific meetings and all our presentations started on the relatively straightforward premise that if we only could unravel the molecular mechanisms of this disease, we would be able to prevent the formation of the disease and thus condemn this disease to the past. I remember that at one such meeting in Europe, an older British surgeon asked why I would ever want to find a non-surgical cure for this disease because Dupuytren's surgery was such an elegant operation to perform and in doing so, surgeons could learn the anatomy of the hand so well. At that time, I did not really know how to answer that question, but the question did stick with me.

\section{New approaches}

Fast forward more than two decades, after countless hours of work (still not leading to the Holy Grail of curing this disease), a much more practical surgeon than me studied the clinical effects of injection of collagenase into the diseased Dupuytren's collagen cords and found that this approach in a certain subset of patients was able to avoid the necessity of extensive surgery previously required to alleviate the contractures. The treatment using collagenase has been hailed as nothing less than a paradigm-changing approach in the treatment of Dupuytren's contracture, with much shorter recovery times and much less need for post-operative rehabilitation. Many patients are very pleased with the collagenase treatment option and commensurately, over a very short time, a sharp drop in the number of Dupuytren's operations occurred. Unfortunately, a sizeable subset of Dupuytren's patients proved to be untreatable using the collagenase option, or they exhibited a rapid and complex recurrence pattern of the disease. In addition, even taking into account the clear advantages of collagenase-treatment in some patients, studies showed that, overall, the gold standard of treatment remained the surgical approach to excise the contracted cords. In group analysis, surgery still results in the best outcome and the lowest recurrence rates but is also the most invasive treatment and requires the most post-operative rehabilitation.

I now teach the collagenase injection to my residents and after years of doing this and observing how my residents inject the disease cords, I came to realize that my residents, who now rarely see an open Dupuytren's operation, actually lack the benefit of a mental picture of what the disease looks like and thus have difficulties deciding where to inject the collagenase. An old-fashioned saying came to mind: "The eye cannot see what the mind does not know"; and I had a flashback to the question of the old British surgeon many years ago.

\section{Unintended consequences}

I realized that many newly-graduating plastic surgeons have never, or hardly ever, done an open Dupuytren's contracture release operation. In the paradox of progress, I now increasingly see patients whose collagenase treatments have failed and who are now being sent to me for treatment-resistant or severe recurrent Dupuytren's disease. These patients often come from far away as local expertise is no longer available for the open treatment of Dupuytren's contracture. It made me think back about the paradox of the great pyramids of Gizeh: Has progress eliminated existing knowledge and skill and, in the case of Dupuytren's, has the advent of a "better" treatment method for a subgroup of patients led to a more difficult treatment algorithm for others and is the use of non-invasive treatment resulting in a general loss of the skills required to surgically treat a disease?

Clearly, this somewhat pessimistic view is not always true. Take, for example, the discovery that peptic ulcer disease, which was traditionally treated with a very invasive partial gastrectomy, vagotomy and or pyloroplasty, was actually caused by infection with the bacteria, Helicobacter pylori. Now peptic ulcer disease is treated with an oral antibiotic and the complex sur- 
gery that was previously performed to treat this disease is merely a foot note in the history of surgery and no one is missing this operation. However, looking back on a surgical career that spans almost three decades, the strange paradox of "progress in one dimension, but loss in another" has occurred in other areas too. The introduction of laparoscopic intra-abdominal surgery in academic centres, with its vastly reduced hospitalization times and lower post-operative morbidity, and the subsequent widespread adaptation of, for example, laparoscopic cholecystectomy in general surgical practice outside academic centres, now mean that difficult laparoscopic cholecystectomies need to be transferred to a university centre for treatment by a hepatobiliary-specialized surgeon for an open cholecystectomy, whereas in the past every general surgeon was able to do this operation. Even the old-fashioned general surgeon is increasingly a relic from the past, and the steep progress in every subarea of general surgery has resulted in a requirement to replace each general surgeon by several subspecialists (colorectal, hepatobiliary, gastro-esophageal, endocrine or breast surgeon) each of whom undoubtedly practices their own subspecialty at a much more advanced and arguably higher and better level, but also one that has lost the knowledge of a large number of procedures that were previously thought to belong in their field of specialty. As another unintended consequence, this super-specialization has resulted in higher overall health care costs and an increase in medical manpower requirements, creating other even more complex problems in our health care system.

In another example of perceived progress, we have seen fundamental research delineating the decline in performance with fatigue. The inference of this research has been that long duty hours could lead to an increase in medical error. In a drive to build a safer health care system, this premise led to marked changes in medical practice, and duty hours became more regulated and restricted. The outcome of these changes was that more people need to be involved in the complex care of patients. Unanticipated in the original studies on the relationship between fatigue and length of duty hours, the logical resulting implementation of duty hour restrictions also led to an increase in patient hand-over, and new data have shown a significant increase in complications and worse clinical outcome parameters associated with just the submission of billing codes associated with patient handover. Whether these data represent true cause-effect relationships or are a pseudo-effect of a reductionist scientific approach remains undetermined and it is unclear whether we have a "better" or "safer" system after the implementation of seemingly logical measures based on apparent solid research.

\section{Ceteris paribus}

Advances in medicine are based on incremental improvements that should, in turn, be based on solid research. The scientific paradigm of reduction to the smallest measurable unit and research under ceteris paribus circumstances direct us to tease out apparently simple direct cause-effect relationships. From these, investigators often infer logical, straightforward solutions to solving the initially-posed scientific problem. But in complex systems such as medicine, is it desirable to look for the straightforward answers? Might we miss the forest for the trees by inferring that reductionist research findings have widespread implications? And as researchers, do we have an obligation to think at a higher level and perhaps have a fuzzy logic-type of approach to medical research and make a stronger effort to anticipate unintended consequences of our research before we proclaim their general validity? How do we recognize, in advance, the hole-in-one changes in our treatment approaches-like the treatment of peptic ulcer disease with antibiotics-that seem to carry no unintended consequence (or perhaps these consequences have not yet been noted) and how do we avoid instituting difficult-to-reverse changes based on seemingly solid research that lead to a one-step-forward/two-steps backward paradox of unintended consequences? Is it possible that by using an over-reductionist approach in research, we go forward in small steps, with the certainty of the correctness of our last steps; but having lost our sense of direction in this approach, we go far in the wrong direction? Is it this kind of research and progress that makes us lose important previously acquired knowledge-our equivalent of knowing how to build pyramids?

\section{CORRESPONDENCE TO:}

E-mail bsgan@rogers.com 